\title{
Feyissa, Dereje \& Hœhne, Markus Virgil (eds.) - Borders and Borderlands as Resources in the Horn of Africa
}

\section{Alain Gascon}

\section{CpenEdition}

\section{Journals}

Édition électronique

URL : http://journals.openedition.org/etudesafricaines/14508

DOI : 10.4000/etudesafricaines. 14508

ISSN : 1777-5353

Éditeur

Éditions de l'EHESS

\section{Édition imprimée}

Date de publication : 16 septembre 2013

Pagination : 740-745

ISBN : 978-2-7132-2388-4

ISSN : 0008-0055

\section{Référence électronique}

Alain Gascon, «Feyissa, Dereje \& Hœhne, Markus Virgil (eds.) - Borders and Borderlands as Resources in the Horn of Africa », Cahiers d'études africaines [En ligne], 211 | 2013, mis en ligne le 20 septembre 2013, consulté le 24 septembre 2020. URL : http://journals.openedition.org/etudesafricaines/14508 DOI : https://doi.org/10.4000/etudesafricaines.14508

Ce document a été généré automatiquement le 24 septembre 2020

(c) Cahiers d'Études africaines 


\title{
Feyissa, Dereje \& Hœhne, Markus Virgil (eds.) - Borders and Borderlands as Resources in the Horn of Africa
}

\author{
Alain Gascon
}

\section{RÉFÉRENCE}

FEYISSA, Dereje \& HÆHNE, Markus Virgil (eds.) - Borders and Borderlands as Resources in the Horn of Africa. Woodbridge-New York, James Currey (« Eastern African Series »), 2010, 224 p., bibl., index.

1 Ce livre est une tentative réussie pour rompre avec les idées reçues qui tiennent, souvent hélas, de grille d'analyse quand les « spécialistes » des médias, commentent les crises africaines: tout commence (et tout finit) en Afrique, par des frontières « injustes ». Les États « importés » enferment dans des frontières artificielles, tracées arbitrairement, des ethnies rivales et, tout au contraire, séparent des groupes homogènes. Ainsi, les limites étatiques, léguées intentionnellement par les colonisateurs, provoqueraient sui generisles conflits ethniques qui pèsent comme une malédiction sur le continent même après un demi-siècle d'indépendance ${ }^{1}$. Cet ouvrage rassemble les contributions de la conférence : « Divided they Stand : The Affordances of State Borders in the Horn of Africa ", organisée par l'Institut Max-Planck, à Halle/Saale du 6 au 8 septembre 2006. D. Feyissa et M. V. Hœhne, les deux directeurs du livre, notent dans leur préface qu'ils ont reçu de nombreuses communications qui insistaient d'abord sur les frontières comme contraintes plutôt que comme ressources. Ils se sont heurtés au prêt-à-penser qui n'est pas réservé aux seuls médias pour qui l'Afrique est malade de ses limites imposées par les colonisateurs. Une fois de plus, on regrette que D. Feyissa et M. V. Hœhne, auteurs du chapitre introductif, "State Borders and Borderlands as Resources. An Analytical Framework», qui ne sont pas anglophones 
mais qui publient en anglais, ignorent les œuvres imprimées dans d'autres langues européennes. Ils ne mentionnent ni F. Ratzel, ni J. Ancel, ni M. Foucher ${ }^{2}$, des auteurs majeurs sur la question des frontières. L'Afrique n'est pas mieux lotie : G. Sautter, M.-C. Acquarone, R. Pourtier et $\mathrm{H}$. Wesseling ${ }^{3}$ sont absents alors que le très convenu ouvrage d'A. I. Asiwaju et P. Nugent ${ }^{4} e s t$ amplement cité, il est vrai pour être épinglé. Les contributions consacrées à la Corne de l'Afrique, toutefois, présentent des bibliographies beaucoup plus diversifiées. Remarquons, et c est rare, que l'éditeur a joint quelques cartes de localisation fort utiles, mais qui ne dispensent pas de recourir aux bonnes vieilles cartes Michelin (Afrique 745 et 746). Comme d'habitude, et c'est une très heureuse coutume, le livre comprend un index et une liste des acronymes où l'on note une erreur fâcheuse: EPRDF ne signifie pas Eritrean People's Revolutionary Democratic Front, mais Ethiopian People's Revolutionary Democratic Front ${ }^{5}$.

2 Les auteurs du chapitre introductif précisent: «The object of the research is how people who live along and are divided by state borders have adjusted to the borderland situation, and what strategies they use in order to extract different types of resources from borders and borderlands » (p. 1). Ils examinent les analyses qui insistent sur le caractère arbitraire des frontières de la Corne, et remarquent qu'après la défaite italienne à Adwa, un acteur africain, l'Éthiopie, s'est imposé comme " producteur » des limites étatiques. Elles ont principalement été tracées dans les basses terres arides, parfois impaludées et qui échappent au contrôle des pouvoirs centraux. Les populations d'éleveurs, qui parcourent les périphéries, y franchissent clandestinement les frontières pour échapper à l'imposition et aux droits de douane et n'hésitent pas à rançonner les commerçants et les agents de l'État et à soutenir des rébellions. Du Kenya, à la Somalie et à l'Érythrée, le terme éthiopien sefta s'est répandu pour stigmatiser ces bandits d'honneur-rebelles-contrebandiers-patriotes que les armées régulières ont traqués, le plus souvent en vain. Enfin, Feyissa et Hœhne constatent que les études n'ont décrit que l'avers du décor et en ont négligé l'envers ou n'ont pas voulu le voir : "In our perspective, it is exactly this lack of precise boundaries that could create opportunities for the "borderlanders" " et notent, ensuite: "The constructedness or arbitrary nature of state borders does not mean they are inconsequential» (p.8). Ils précisent plus loin: «[...] We reiterate that we are interested not in what the borders have done to the people, but in what the people have done to the borders, and in what they have made out of living in the borderlands as fields of opportunities» (p.11). Ils se heurtent, également, au sentiment de victimisation associé à l'Afrique et qui est bien commode pour dégager les dirigeants africains de leur responsabilité dans la perpétuation des troubles affectant le continent et sa péninsule la plus orientale. Dans la Corne, à l'instar du Tchad ou de l'Ouganda, on note que les révoltes, nées aux confins, ont conquis le pouvoir central à l'instar les fronts érythréens et tegréen qui s'installèrent à Asmara et à Addis Ababa (1991). Les rébellions n'ont aucunement voulu effacer les frontières mais, bien au contraire, comme en Érythrée, au Somaliland et au Sud-Soudan ${ }^{6}$, restaurer des limites coloniales officiellement honnies. Que deviendraient les frontaliers qui vivent du transbordement, illégal et légal, entre Djibouti et Dirré Dawa et entre le Harar et le Somaliland ${ }^{7}$ si ces limites s'effaçaient? Dans la seconde partie de leur introduction, Feyissa et Hœhne présentent les communications à venir dans les perspectives de l'analyse qu ils viennent d'exposer. On pourra lire, à la suite, la remarquable conclusion « Putting back the Bigger Picture» (pp. 187-195), rédigée par C. Clapham qui complète cette introduction. 
3 Présenter un compte-rendu d'un ouvrage de cette importance n'est pas aisé car chacun des huit chapitres mériterait, en lui-même, une recension. Je mentionnerai donc toutes les contributions même si le lecteur trouve ce procédé un peu fastidieux. On peut, toutefois, rassembler les textes traitant des peuples à cheval sur la frontière qui luttent pour se faire une place au centre, à l'échelle nationale, et s'appuient sur leurs " frères » d'au-delà des limites afin d'obtenir un abri, des subsides et des armes (Fekadu, Yasin). Une autre stratégie des frontaliers est d'offrir, moyennant rétribution et promotion, ses services à l'État qui cherche à contrôler ses confins : on se rallie aux plus offrants, suivant la conjoncture (Hœhne, Barnes). En tenant les échanges commerciaux, les frontaliers peuvent tirer bénéfice, dans un sens comme dans l'autre, des différentiels de prix et de tarifs et des fluctuations de la conjoncture entre États voisins (Cassanelli, Wafula). Les autres contributions présentent des cas plus complexes ou plus particuliers en dehors des basses terres orientales de la Corne de l'Afrique (Dereje, Smidt, Declich).

4 Fekadu Adugna, «Making Use of Kin beyond the International Border. Inter-ethnic Relations along the Ethio-Kenyan Border » et Yasin Mohammed Yasin, «Trans-Border Political Alliance in the Horn of Africa. The Case of the Afar-Issa Conflict » étudient comment les gouvernements centraux coloniaux et postcoloniaux (lors de l'occupation italienne de l'Éthiopie) ont choisi d'appuyer les revendications territoriales d'un groupe de population en échange de la garde de la frontière. Ils montrent les permutations d'alliance qui ont accompagné les changements politiques au centre. Ainsi, les gouvernements éthiopiens, de Menilek à Mangestu, ainsi que les Italiens ont-ils cherché à gagner les Oromo qui subissaient l'avancée des Somali au Kenya. Depuis qu'en 1992, le Front de libération des Oromo (FLO) a quitté la coalition du FFRPE, dirigée par Mälläs, le gouvernement fédéral éthiopien a revu son soutien exclusif pour se tourner vers les peuples de la région-État(kellet)multinationale «Sud» créée pour faire pièce au kellel Oromiyaa. Les Afar, dont la majorité réside en Éthiopie dans un kellel autonome, ont subi le contre-coup de la sécession érythréenne et de la montée des ambitions nationales, et internationales, du président de Djibouti, Ismaël Omar Guelleh (IOG). Jusqu'aux émeutes indépendantistes qui accueillirent le général de Gaulle, lors de son escale vers Addis Abeba en 1966, l'administration de la Côte française des Somalis négligeait les Afar qu'elle redécouvrit en donnant le gouvernement du Territoire français des Afars et des Issas (TFAI) à Ali Aref Bourhan, en Afar. En 1977, la république de Djibouti devint indépendante, présidée par Hassan Gouled Aptidon, un Somali Issa (Ciise). La révolution réunit les Afar d'Éthiopie et d'Érythrée dans une seule région à laquelle elle confia la surveillance du port et de la route d'Asäb. Avec la chute de Mängestu en 1991, les deux gouvernements brisèrent toute velléité d'union chez leurs Afar tandis qu'IoG, ministre de l'Intérieur, aidé par la France, réprimait la révolte des Afar. Devenu président, il soutient, avec de l'armement de contrebande, les Somalis d'Éthiopie qui repoussent les Afar vers l'Awash. Il se pose en champion de l'unité somalienne dans le nord de la Corne, face à la sécession du Somaliland dont le port, Berbera, menace l'hégémonie de Djibouti. Ces deux études montrent comment les pouvoirs centraux utilisent les populations frontalières non seulement pour les garder, mais pour poursuivre au-delà des frontières leurs objectifs géopolitiques régionaux.

5 Les contributions de Markus V. Hœhne: "People and Politics along and across the Somaliland-Putland Border » et de Cedric Barnes : "The Ethiopian-British Somaliland Boundary" ont en commun d'être consacrées au Somaliland. Elles examinent un 
rapport de force qui avantage les frontaliers : ils ne sont plus les clients des États. Au vu de ce qu'on leur propose (imposition, équipements, carrières), ils choisissent tel ou tel gouvernement, libres à eux s'ils trouvent meilleure offre ailleurs. Ces textes vont également à l'encontre des idées reçues qui dépeignent les Somalis comme d'éternelles victimes du partage colonial entériné par l'ouA.Barnes révèle combien les Éthiopiens se sont appuyés sur les Somalis agriculteurs, de «bons» contribuables, alors que les Britanniques soutenaient les éleveurs tout en instrumentalisant les clans et les confréries. Hœhne étudie de façon très convaincante les carrières d'officiers et de fonctionnaires qui oscillent entre Somaliland et Puntland au gré des avantages que leur proposent les deux administrations, au besoin en «bricolant » les attaches cla-niques et confrériques. Il rejoint ainsi les travaux de M. Djama et l'affirmation de Nurrudin Farah : Clan Business, clan mafia 8 .

6 Le cloisonnement de l'espace économique apparaît comme l'un des reproches les plus graves opposés aux frontières coloniales. Elles ont entravé les échanges et porté le coup de grâce au commerce interafricain au profit de la préférence coloniale. Les textes de Lee Cassanelli, "The Opportunistic Economies of the Kenya-Somali Borderland in Historical Perspective» et de Peter Wafula Wekesa, "Magendo and Survivalism. Babukusu-Bagisu Relations and Economic Ingenuity on the Kenya-Uganda Border 1962-80» montrent combien les échanges persistent en dépit des frontières, des guerres et des répressions les plus brutales menées par les dirigeants. Jamais Idi Amin Dada n'a réussi à interrompre le magendo (la contrebande) à la frontière kenyoougandaise qui a permis la survie des populations. Cassanelli remonte dans le temps long, d'avant la période coloniale, et souligne combien les routes commerciales entre l' hinterland et les ports du Benaadir ont résisté au compartimentage territorial contemporain.

7 Les études dont il sera question combinent, en quelque sorte, les trois « utilisations » des territoires frontaliers et des frontières décrites plus haut. Dans « More State than the State? The Anywaa's Call for the Rigification of the Ethio-Sudanese Border », Dereje Feyissa retrace comment les rivalités séculaires entre Nuer et Anuak (Anwaa), dans cette marge oubliée de l'ouest de l'Éthiopie, le kellel de Gambéla, ont pris une dimension régionale et nationale. La guerre dans le sud du Soudan a entraîné la migration massive de Nuer, agroéleveurs, dans des camps situés en territoire éthiopien car le diirg, la junte, soutenait le SPLM $/ \mathrm{A}^{9}$ de John Garang alors que Khartoum aidait les fronts érythréens et tegréen. Les Anuak, agro-éleveurs sédentaires, déjà moins nombreux, se sentirent alors dépossédés par les réfugiés qui bénéficiaient, en outre, de l'aide des ONG. Soutenus par l'EPRDF/FDRPE, les Anuak, alliés aux autres peuples minoritaires et renforcés par l'afflux des populations de hautes terres, dominent le gouvernement régional et demandent instamment aux autorités centrales de bloquer les "Soudanais » (i.e.: les Nuer) à la frontière. Cette connivence des Anuak et du gouvernement fédéral, nouée au cours de la guerre contre l'Érythrée, va-t-elle survivre à la ruée sur les basses terres entamée en 2009 avec l'octroi de centaines de milliers d'hectares, pour dix, vingt et trente ans, à des firmes agro-industrielles indiennes et éthio-saoudiennes, produisant pour l'exportation ${ }^{10}$ ?

Wolbert W. C. Smidt aborde dans «The Tigrinnya-speakers across the Borders. Discourse of Unity Separation in Ethnohistorical Context » la question brûlante de la frontière éthio-érythréenne, le long de laquelle plusieurs centaines de soldats se sont battus entre 1998 et 2000. On a largement qualifié d'absurde ce conflit, entre deux 
anciens alliés, pour une limite coloniale qui divise, en son milieu, un groupe linguistique homogène ayant fait partie de l'Éthiopie historique jusqu à la colonisation italienne de l'Érythrée en 1890 et dont la partie érythréenne a été fédérée puis annexée à l'Éthiopie de 1952 à 1991. Le grand talent de l'auteur, tour à tour ethnologue, linguiste et historien, se déploie dans une démarche multiscalaire. Il identifie deswe groups,des groupes où l'on dit nous, qui se combinent?, s'allient et s'opposent tout au long de l'histoire récente. Dans ces groupes on retrouve, en proportion quasi égale, des arguments pour l'unité et pour la séparation: "A common Tigrinnya identity has always opened up two possibilities : to follow a common Tigrinnya project and/or local interests and alliances » (p. 78). Ainsi, W. Smidt affirme-t-il, et on le suit volontiers, que les locuteurs du tegrenna (tigrinnya) ne forment pas un "bloc ethnique», que la frontière n'est pas née avec la colonisation, qu elle fut mouvante et que sa reconnaissance internationale est une chance pour que la stabilité s'installe dans la région. Le texte de Francesca Declich «Can Boundaries not Border on One Another? The Zigula (Somali Bantu) between Somalia and Tanzania " traite de populations à peu près ignorées, les Bantous des vallées inférieures, bordées de forêts-galeries, du Wabi Shabeele et du Jubba. Elle nous rappelle qu'ils furent, même après l'indépendance, considérés comme les hors-clans, les forgerons et les tanneurs comme des Somaliens de seconde classe car assimilés à des descendants d'esclaves et donc esclaves. La dictature de Siyaad Barre abolit ces distinctions qui resurgirent avec la guerre civile qui éclata en 1988 et se poursuit jusqu'à maintenant. De guerre lasse, les Zigula sont repartis en nombre en Tanzanie d'où leurs ancêtres étaient venus, il y a quelques siècles et le gouvernement tanzanien vient d'en naturaliser plus de 50000 ! Encore un chapitre à verser à la persistance de l'esclavage en Afrique, dans une Afrique où ni les Européens ni les Arabes les transportèrent.

9 Par sa problématique originale et par ses études novatrices et précises, cet ouvrage renouvelle la géopolitique et l'histoire des frontières dans la Corne de l'Afrique et même bien au-delà. Va-t-il porter un coup décisif aux lamentations sur la malédiction des frontières qui aurait fondu comme une macule sur le continent et à la mode du « sans frontières $»^{11}$ ? On l'espère, mais on en doute.

\section{NOTES}

1. Voir ma contribution, A. GASCON, «La mauvaise réputation des frontières africaines », in $D$. PAPIN(dir.), 50 fiches pour comprendre la géopolitique, Paris, Bréal, 2010, pp. 78-81.

2. F. RATZEL, Politische Geographie,Munich-Berlin, R. Oldenbourg, 1903 ( $2^{\mathrm{e}}$ éd.), (traduction P. Ruch, Géographie politique, Genève, Éditions régionales européennes ; Paris, Économica, 1988) ; J. ANCEL, Géographie des frontières, Paris, nrf, Gallimard, 1938 ; M. FoucHER, Fronts et Frontières, un tour du monde géopolitique, Paris, Fayard, 1988.

3. G.SAUTTER, « Quelques réflexions sur les frontières africaines ", Pluriel, 30, 1982, pp. 41-50 ; M.-C. AQUARONE, Les frontières du refus: six séparatismes africains, Paris, CNRS, 1987 ; R. POURTIER(dir.), Géopolitique de l'Afrique et du Moyen-Orient, Paris, Nathan, 2006 ; H. WESSELING,Le partage de l'Afrique 1880-1914, Paris, Denoël, 1991 (Folio-Histoire, 1996). 
4. African Boundaries. Barriers, Conduits and Opportunities, London-New York, Pinter-Centre of African Studies, University of Edinburgh, 1996. Voir mon compte rendu paru dans les Cahiers d'Études africaines, 158, 2000.

5. L'EPRDF (FDRPE : Front démocratique et révolutionnaire du peuple éthiopien) est une coalition de partis au pouvoir depuis 1991, dirigée par le Front populaire de libération du Tegray du Premier ministre, Mälläs Zénawi.

6. La nouvelle frontière, entre le Nord et le Sud, suit les limites des Restricted Districts,interdits aux Égyptiens par les Britanniques, au temps du Soudan anglo-égyptien.

7. M. DJAMA, «Trajectoire du pouvoir en pays somali », Cahiers d'Études africaines, XXXVII (2), 146, 1997, pp. 349-377.

8. Lors d'une conférence à l'EHESS en 2006.

9. Sudan People's Liberation Movement/Army : au pouvoir depuis 2005, à Juba, au Sud.

10. Voir ma communication "Màrét làfàràngu [la terre aux étrangers] : la ruée vers la terre en Ethiopie (et au Soudan) »,Intorno a Orazio Antinori Pensieri e pratiche della natura africana dalle visioni coloniali allo sviluppo locale sostenibile, Rome, Società Geografica Italiana, 7-8 juin 2011.

11. A. SABATIER, «Petite philosophie de la frontière »,<http://www.lrdb.fr/articles.php? lng=fr\&pg=92>, mis en ligne en décembre 2006. 